\title{
MAGNETOHYDRODYNAMICAL PHENOMENA IN THE SOLAR CORONA AS REVEALED BY YOHKOH
}

\author{
YUTAKA UCHIDA \\ Department od Physics, Science University of Tokyo, Shinju-ku, Tokyo 162 \\ Department of Astronomy, University of Tokyo, Bunkyo-ku, Tokyo 119
}

\section{Introduction}

In the talk, some of the new findings about the magnetodynamical phenomen a in the inner corona obtained by the satellite Yohkoh were reviewed. Here we give a very brief summary of it. (The long list of references has been omitted due to the limitation of space here.)

One of the most remarkable findings by Yohkoh is that the corona is quite a dynamic entity. Such a dynamicity was recognized in its full form by the wide-dynamic range, high cadence imaging from Yohkoh-Soft X-ray Telescope (SXT) with the use of the video-movie presentation. It turned out that the background corona formed quite a dynamic system with the active regions (Uchida 1992, Tsuneta and Lemen 1992), and many examples of interesting dynamic interplay between them were found. It may well be said that the corona is not an "atmosphere" of the usual sense, but is an entity highly electromagnetic in character.

The dynamic behavior of the corona found by Yohkoh was predominantly seen (i) in relation to the disappearance of dark filaments associated with the magnetic field-polarity-reversal lines in the photosphere, either in active regions (then, an arcade flare), or in the region far from active regions (then a fainter arcade formation), or (ii) in relation to very small changes in strong field active regions influencing a very large region of the corona with weak field.

\section{X-ray Arcade Formation and Arcade Flares}

The disappearance of $\mathrm{Ha}$ dark filament outside active regions was known to cause an extended low-energy $\mathrm{H} \alpha$ double-ribbon brightenings. Detailed dynamic behavior of X-ray arcade formation above them has been made clear by Yohkoh. Some of the new findings (McAllister et al. 1992, Uchida et al. 1995) seem to be difficult to explain in the classical models discussed thus far, and suggest the necessity of more satisfactory models: A very instructive example is the Feb 21, 1992 limb flare. Tsuneta et al. (1992a) have given an analysis of 
this flare, covering from its pre-flare state to the post-flare state, and found that the morphology of this flare at its maximum seemed to be consistent with the classical model (namely, the dark filament rise opens up the arcade field, and the reclosing of the thus cut up field through magnetic reconnection liberates energy, and evaporates the chromospheric plasma to fill the structure , causing the $H \alpha$ double-ribbon flare below (Sturrock 1964, Hirayama 1974, Kopp and Pneuman 1976, Priest and Forbes 1990). The preflare magnetic structure revealed by Yohkoh, however, showed a connectivity indicating that quadrupolar field source was involved, not a bipolar one as assumed in the classical model. Another well observed arcade type flare at the limb, Dec 2, 1991 flare, had this, too. Quadrupolar source model was proposed for the magnetic configuration of dark filament suspension (Uchida and Jockers 1979), and a flare model inherent to this was discussed to solve some of the difficulties of the conventional models of flares by the present author (Uchida and Sakurai 1980). Those models, however, were not received too well that time. Those models considering a quadruple source are being revived since observations by Yohkoh revealed features suggestive of them (Uchida et al. 1995).

A larger version of the arcade formation occurring in high latitude zones far from the active latitude was observed by Yohkoh on Nov 12, 1991, with the progressive formation of $3 \times 10^{5} \mathrm{~km}$ height and $10^{6} \mathrm{~km}$ long (Tsuneta et al. 1992b). The appearance of a large cusp-like feature on the south-west limb on Jan 25, 1992 was noted as a feature related to the coronal mass ejection (CME) and was considered to be not incompatible with the classical picture (Hiei et al. 1993). Here again, a closer comparative examination with the similar event of Feb 25, 1993 indicates some features seriously contradicting the expectation from the classical model. For example the two feet of the "cusp" turned out to be still separated by tens of degrees in the heliographic longitude from each other after formation (Uchida et al. 1995b in preparation).

\section{Behavior of Active Regions and the Changes in the Large Scale Corona Associated with Them}

The conventional concept of the active region corona, a region of a hot and dense gas tied down by the sunspot magnetic field to the photosphere with some smooth heating process, turned out to be not the case. Active region corona is found to be supplied with the already heated mass from below along the magnetic loops (Shimizu et al. 1992). Also, the active region corona expanding in many cases almost continually (Uchida et al. 1992), with a velocity of the order of $10 \mathrm{~km} / \mathrm{s}$, showing that it is not in magnetohydrostatic equilibrium as has been considered. It seems that these are the prototypes of the mass (and current) injection into a part of the flux tube systems, and destabilization of the part 
of the system. We see heated mass ejection from a small bright point in active regions into the magnetic structures surrounding the active regions, and in some cases a brightening of a large region of the corona is induced by that, and in the Apr 27, 1992 event, a large arcade formation was caused by that at a distance of $4 \times 10^{5} \mathrm{~km}$. This shows that a small brightening in active regions can disturb a wide region of the corona, and sometimes can cause CME's. This can solve the paradox raised by Harrison about the causality between the flare and CME.

\section{Global, Cycle-phase Dependent Phenomena, like Coronal Holes and X-ray Bright Points}

Skylab found an enigmatic behavior of a coronal hole executing a rigid-body rotation while all other surface signatures showed differential rotation. Some people thought that this behavior of the coronal hole might be related to the behavior of deep-seated magnetic field, and might reveal the behavior of dynamo process in the interior of the Sun. Some of the synoptic analyses of the Yohkoh data, however, revealed that the high latitude parts of the coronal hole $s$ were not rotating rigidly, and the rigid rotation of the coronal hole was restricted only for the part in the active latitude zones (Takahashi et al.1993). The rigid rotation of the coronal holes claimed by the Skylab result might be a reflection of the rigidly rotating active longitude belts.

The behavior of the X-ray bright points was examined in Skylab (Golub et al.1974), and examined with Yohkoh (Strong et al.1992, Nitta et al.1992), but the number of the XBP is considerably less, and do not show a marked cycle phase dependence thus far.

The author acknowledges the Japanese, US, and UK core members of Yohkoh and the staff of ISAS for making this wonderful satellite Yohkoh possible, and the Team Members for good observation and analysis of the data. 